\title{
Análisis estadístico de la velocidad de operación de vehículos pesados en pendientes ascendentes
}

\author{
Statistical analysis of heavy vehicle operating speed in ascending slopes
}

Fecha de entrega: 4 de marzo 2015 Fecha de aceptación: 24 de agosto 2015

\section{Tomás Echaveguren y Daniela Arellano}

Departamento de Ingeniería Civil, Universidad de Concepción, Edmundo Larenas 219-interior, Casilla 160-C, Correo 3, Concepción, Chile, techaveg@udec.cl, danarellano@udec.cl

El perfil de velocidad de los vehículos pesados en pendientes ascendentes se utiliza para proyectar pistas auxiliares. Las normativas consideran un camión con una relación peso/ potencia típica, una velocidad fija de ingreso a la pendiente, pendientes uniformes hasta $8 \%$ y longitudes de pendientes hasta $12 \mathrm{~km}$. Estos perfiles se obtienen mediante el equilibrio de las fuerzas que se oponen al movimiento y la fuerza del motor del vehículo pesado. Experimentos realizados en Alemania muestran que los perfiles de velocidad reales difieren de los modelados, por lo cual es pertinente analizar en terreno el comportamiento de los vehículos pesados en las pendientes ascendentes. En este trabajo se presenta un estudio empírico de perfiles de velocidad de vehículos pesados en pendientes ascendentes, a fin de contrastar la evidencia empírica con el modelo provisto por la normativa Chilena. Se seleccionaron 24 tramos de medición con pendientes entre $2 \%$ y $13 \%$ y entre 0.2 y $2.4 \mathrm{~km}$ de longitud. Se obtuvieron 70 perfiles de velocidad mediante un GPS cinemático. Los datos se corrigieron usando el filtro de Kalman para posteriormente aplicar regresión no paramétrica y obtener perfiles de velocidad continuos. Se analizó estadisticamente las velocidades de entrada, máxima, minima y de salida, la forma del perfil y su relación con la normativa Chilena. Se concluyó que los modelos sobre-estiman la caída de velocidad en la primera parte de la pendiente, no consideran el efecto de aceleración en la cima de la pendiente y no siempre se alcanza la velocidad de equilibrio.

Palabras clave: velocidad de operación, vehículo pesado, pendiente ascendente
The truck speed profiles in ascending slopes are used to design climbing lanes. The standards consider a vehicle with typical weight-to-power ratio, uniform slopes up to $8 \%$ and slope lengths of $12 \mathrm{~km}$. The speed profile is obtained equating the forces opposed to the movement and the forces provided by the truck engine. Experiments conducted in Germany show that the actual speed profiles are different from that of theoretical speed profiles. Therefore, it is relevant to study in the field the truck speed behaviour in ascending slopes. This paper presents an empirical study of truck speed profiles in ascending slopes. The study characterizes and compares measured speed profiles with a theoretical model used in the Chilean geometric design standards. A set of 24 test sections, with slopes between $2 \%$ and $13 \%$ and lengths between 0.2 and $2.4 \mathrm{~km}$ were used. 70 speed profiles using a kinematic GPS device were obtained. Data were corrected using the Kalman filter and smoothed using non-parametric regression to obtain continuous speed profiles. The entrance speed, the maximum and minimum speed and the shape of the speed profiles were statistically analysed as well as the relationship between these parameters and the parameters used in the Chilean standard. It is concluded that the theoretical model overestimates the speed reduction in the first part of the slope, it does not consider the acceleration at the end of the slope and that the trucks not always reduce the speed up to the crawl speed.

Keywords: operating speed, heavy vehicle, ascending slope

\section{Introducción}

El perfil de velocidad representa la variación de la velocidad de un vehículo pesado a medida que avanza por un tramo de carretera. Las normas de diseño geométrico de carreteras utilizan este tipo de perfiles en pendientes ascendentes para evaluar la necesidad de proyectar pistas auxiliares 
para vehículos pesados, bajo criterios de seguridad y de nivel de servicio. Tales normativas utilizan perfiles basados en un vehículo pesado tipo, con una potencia típica, con una velocidad de ingreso a la pendiente, una pendiente uniforme entre 0 y $12 \%$ y longitudes que varían entre 0 y $6 \mathrm{~km}$. Los perfiles de velocidad se obtienen en base a modelos mecanicistas que estiman la velocidad del vehículo en función del equilibrio entre la fuerza tractriz del vehículo pesado y las resistencias al movimiento en planos inclinados. Uno de los modelos más conocidos en la literatura es el de Rakha et al. (2001), el cual permite determinar perfiles de velocidad para cualquier pendiente, cualquier longitud y diversas relaciones entre el peso del vehículo y su potencia, a este modelo, Rakha y Lucic (2002) le agregan el efecto del cambio de marcha. Normalmente estos perfiles de velocidad son monótonos decrecientes, de modo tal que la velocidad al inicio de la pendiente decrece progresivamente hasta llegar a una velocidad de equilibrio que se mantiene hasta el final de la pendiente.

Si bien los perfiles de velocidad estandarizados son adecuados por su simplicidad, en la práctica la variabilidad de la pendiente longitudinal y los cambios de marcha de los vehículos pesados inducen distorsiones en los perfiles de velocidad que los modelos mecanicistas no representan. Los experimentos de Verweij (2000) realizados en Alemania corroboran lo anterior. Verweij obtuvo perfiles de velocidad en los cuales la velocidad inicial se incrementaba ligeramente en el inicio para luego descender, alcanzar una velocidad de equilibrio y posteriormente aumentar antes del término de la pendiente. Este comportamiento dista de aquel evidenciado por los modelos. El uso de camiones instrumentados como en el experimento de Verweij (2000) constituye una alternativa adecuada para obtener perfiles de velocidad, por cuanto es posible controlar diversas variables de operación, tales como el peso y la potencia. Sin embargo, limita notablemente el tamaño de la muestra a emplear, restándole generalidad a la investigación. Por otro lado, el uso de técnicas convencionales de medición de velocidad de operación basadas en mediciones puntuales otorga sólo unos pocos puntos de medición, lo cual en pendientes largas limita también el tamaño de la muestra. Asimismo, limita la posibilidad de obtener en terreno la relación entre el peso y la potencia del vehículo medido, variable necesaria para calibrar modelos de perfiles de velocidad.

En Chile, las instrucciones de diseño utilizan un perfil único de velocidad para vehículos pesados en pendientes ascendentes, adoptado de la normativa de Estados Unidos, el cual se expresa en el Manual de Carreteras (MOP 2010) en forma gráfica. Hasta ahora, no se han desarrollado estudios para verificar la validez de dicho perfil de velocidad. En tal sentido, este trabajo tiene por objetivo estudiar en terreno el comportamiento de los vehículos pesados en pendientes ascendentes. Para ello se realizó un estudio empírico aprovechando las opciones tecnológicas que ofrecen los sistemas GPS dinámicos. Para ello se utilizó un GPS dinámico sub-métrico con precisión de $0.1 \mathrm{~km} / \mathrm{h}$, el cual se dispuso en un vehículo liviano para obtener velocidades mediante seguimiento vehicular. Este dispositivo permitió capturar velocidades de operación cada $0.1 \mathrm{~s}$. Se seleccionaron 24 tramos de medición con pendientes ascendentes variables entre 2 y $13 \%$ y entre 0.2 y $2.4 \mathrm{~km}$ de longitud todos ellos localizados en la cordillera de la costa en la zona centro sur de Chile. Se obtuvieron 70 mediciones de perfil de velocidad, usando la técnica de seguimiento vehicular. Posteriormente los datos de velocidad se procesaron aplicando en primer lugar una corrección de velocidad aplicando el modelo de Gaziz et al. (1961), para luego aplicar el filtro de Kalman disponible en el software del equipo GPS, con la finalidad de eliminar datos anómalos y acoplar las mediciones de posición y de velocidad. Luego se aplicaron técnicas de suavización de datos para comprimir los datos y eliminar el ruido de la señal ocasionado por pequeñas variaciones de velocidad que registra el GPS. Con esto se obtuvieron perfiles continuos de velocidad, los cuales se analizaron y compararon con los propuestos por la normativa de diseño de Chile.

Con los resultados de esta investigación, se pretende contribuir a la comprensión del comportamiento de vehículos pesados en pendientes descendentes sobre la base de datos empíricos, lo cual permite crear una base de conocimiento que permita en el futuro incorporar a las normas de diseño geométricos de carreteras perfiles de velocidad para camiones más realistas y representativos de la flota nacional de vehículos pesados. 


\section{Los perfiles de velocidad de camiones en las pendientes}

\section{El fenómeno físico}

Existen dos tipos de perfiles de velocidad en pendientes uniformes $i$, dependiendo del signo de la pendiente longitudinal. En pendientes ascendentes $(i>0)$ los vehículos tienden a disminuir la velocidad, $\mathrm{y}$ en pendientes descendentes $(i<0)$, tienden a aumentarla. A medida que avanzan por la pendiente, los vehículos en las dos situaciones logran una velocidad de equilibrio bajo condiciones de control, representada por los tramos CE y DF de la Figura 1 (las distancias y velocidad son referenciales). El perfil de velocidad de un vehículo pesado en una pendiente, tiene dos tramos característicos. En el primer tramo (BD o AC) el vehículo se encuentra acelerando o desacelerando dependiendo si se encuentra en pendiente ascendente o descendente. Luego en el segundo tramo (CF o DE) la velocidad tiende a mantenerse constante, ya sea por equilibrio de fuerzas o por la aplicación de frenos. El análisis de la velocidad de camiones en pendientes descendentes no se discute en este trabajo. Sin embargo puede revisarse en Echaveguren y Vargas (2007).

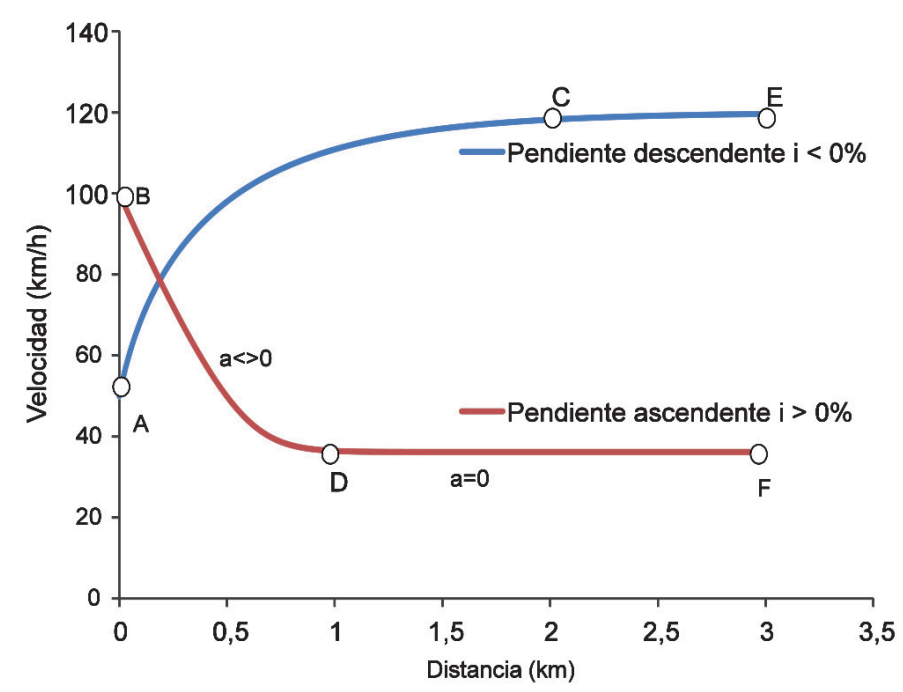

Figura 1: Perfil de velocidad en pendientes uniformes

En pendientes ascendentes la velocidad disminuye desde un valor inicial (punto B) hasta una velocidad de equilibrio (punto D). Esta desaceleración ocurre debido al aumento de las fuerzas resistivas frente a la fuerza proporcionada por el motor (Rakha y Yu, 2004). Al equilibrarla, la velocidad permanece constante. Dicha velocidad se denomina velocidad de equilibrio (crawl speed) y corresponde a la máxima velocidad que puede alcanzar un vehículo pesado que circula por una pendiente longitudinal en ausencia de aceleraciones o deceleraciones. Su magnitud y la distancia a la que se alcanza (punto C o D de la Figura 1) depende de: la longitud del tramo en subida, la inclinación de la pendiente ascendente, la razón peso/potencia $(\mathrm{P} / \mathrm{P})$ del vehículo, la altura sobre el nivel del mar, la velocidad inicial del vehículo, la presencia de curvas horizontales, de restricciones laterales y la visibilidad (Fitch, 1994; Archilla y Fernández de Cieza, 1996; Wong, 2001).

\section{Aspectos normativos}

Debido a la necesidad de estandarizar el comportamiento de los camiones en pendientes ascendentes, las normas de diseño geométrico utilizan perfiles de velocidad estandarizados. Las normativas de Estados Unidos (AASHTO, 2011), España (DGC, 1999), Australia (AUSTROADS, 2009), Chile (MOP, 2010) y Colombia (INVIAS, 2008) representan una buena muestra de las prácticas normativas a nivel mundial. Las normas revisadas utilizan el perfil de velocidad de vehículos que enfrentan una pendiente ascendente para definir las pendientes máximas permitidas, la longitud máxima de la pendiente y para verificar si es necesario proyectar una pista exclusiva de ascenso para vehículos pesados. En la Tabla 1 se muestra un resumen con las principales variables de los perfiles de velocidad de las normas anteriormente revisadas: la relación $\mathrm{P} / \mathrm{P}$, la velocidad de entrada $\mathrm{y}$ valor de la pendiente y el rango de pendientes. Estos 4 parámetros de modelación exhiben una amplia variedad entre normativas, la cual depende de las condiciones geográficas y de las características de la flota de vehículos pesados de cada país.

La razón $\mathrm{P} / \mathrm{P}$ que utilizan las normativas corresponde a un vehículo pesado típico. Cabe preguntarse entonces cómo se relaciona la evidencia empírica con los modelos teóricos propuestos por las normativas de diseño. En particular, AUSTROADS (2009) utiliza 4 perfiles de velocidad para 4 tipos de vehículos pesados. En la Tabla 1 se presenta el vehículo que es análogo a las otras normas para efectos de comparación. 
Tabla 1: Resumen de variables descriptivas de perfiles de velocidad de vehículos pesados tipo en pendientes ascendentes

\begin{tabular}{|l|c|c|c|c|}
\hline $\begin{array}{l}\text { País de } \\
\text { origen de la } \\
\text { normativa }\end{array}$ & $\begin{array}{c}\text { Peso/ } \\
\text { Potencia, } \\
\mathrm{kg} / \mathrm{kW}\end{array}$ & $\begin{array}{c}\text { Veloc. de } \\
\text { entrada, } \\
\mathrm{km} / \mathrm{h}\end{array}$ & $\begin{array}{c}\text { Longitud } \\
\text { de la } \\
\text { pendiente, } \\
\mathrm{km}\end{array}$ & $\begin{array}{c}\text { Rango de } \\
\text { pendien- } \\
\text { tes, } \%\end{array}$ \\
\hline $\begin{array}{l}\text { Estados Uni- } \\
\text { dos } \\
\text { (AASHTO, } \\
\text { 2011) }\end{array}$ & 120 & 110 & $<6.0$ & $1-9$ \\
\hline $\begin{array}{l}\text { Chile } \\
\text { (MOP, 2010) }\end{array}$ & 122 & 88 & $<3.9$ & $1-8$ \\
\hline $\begin{array}{l}\text { Australia } \\
\text { (AUSTRO- } \\
\text { ADS, 2009) }\end{array}$ & 103 & 100 & $<1.8$ & $1-10$ \\
\hline $\begin{array}{l}\text { Colombia } \\
\text { (INVIAS, } \\
\text { 2008) }\end{array}$ & 148 & 90 & $<2.0$ & $3-9$ \\
\hline $\begin{array}{l}\text { España } \\
\text { (DGC, 1999) }\end{array}$ & $\begin{array}{c}\text { No dispo- } \\
\text { nible }\end{array}$ & 80 & $<2.4$ & $2-8$ \\
\hline
\end{tabular}

Modelos de perfil de velocidad de camiones en pendientes ascendentes

Estos modelos se agrupan en tres tipos: los basados en el movimiento cinemático, como el de Lee y Lee (2000); los que incorporan conceptos de dinámica del vehículo como los de Gillespie (1985) y Rakha et al. (2001); y los empíricos, como el de Verweij (2000). Los modelos cinemáticos describen el movimiento del vehículo mediante el desplazamiento, velocidad y aceleración, sin considerar los factores que producen el movimiento. Por ejemplo, Lee y Lee (2000) utilizaron el principio de conservación de la energía combinado con variables cinemáticas para obtener la velocidad del vehículo en cualquier punto de la pendiente. La expresión (1) muestra en términos generales el modelo de Lee y Lee (2001) para tramos de pendientes uniformes ascendentes, en donde $V$ es la velocidad en el tramo con pendiente $i$ constante; $m$ es la masa del vehículo, $L_{\mathrm{e}}$ es la distancia a la cual el vehículo alcanza la velocidad de equilibrio $V_{\mathrm{e}}$. La expresión (2) representa el mismo modelo para el tramo de pendiente ascendente variable $i(x)$. $L$ representa la distancia total de la pendiente.

$$
\begin{aligned}
& \frac{1}{2} m V^{2}+\left(m \frac{d V}{d t}\right) L_{e}=\frac{1}{2} m V_{e}^{2}+(m g) i L_{e} \\
& \frac{1}{2} m V^{2}+(m g) i L_{e}+\left(m \frac{d V}{d t}\right)\left(L-L_{e}\right)= \\
& \frac{1}{2} m V_{e}^{2}+(m g) i L
\end{aligned}
$$

Los modelos dinámicos describen el comportamiento de la velocidad del vehículo pesado en base a la fuerza tractiva, la resistencia aerodinámica, la resistencia al rodado y la resistencia por pendiente. Varios modelos han sido desarrollados en base a este método, siendo el de Rakha et al. (2001) el más influyente. Lan y Menendez (2003) incorporaron además elementos de la cinemática para dar solución a la variación de la aceleración en el perfil de velocidad durante el ascenso.

La expresión (3) representa el modelo de Rakha et al. (2001) basado en equilibrio de fuerzas. Por otro lado, la expresión (4) corresponde a la solución discreta de la ecuación diferencial, que permite obtener el perfil de velocidad en función de la distancia a partir de (3). $P$ corresponde a la potencia efectiva del vehículo pesado y $\alpha_{i}$ son coeficientes del modelo. La expresión detallada puede verse en Rakha y Yu (2004).

$\frac{d V}{d t}=\frac{F_{T}-R_{A}-R_{R}-R_{G}}{m}=$

$\frac{\max \left\{\alpha_{1} \frac{P}{V}, F_{M A X}\right\}-\alpha_{2} V^{2}-\alpha_{3}\left(\alpha_{4}+\alpha_{5} V\right) m-m g i}{m}$

$\frac{d V\left(t_{i}\right)}{d t}=\frac{F_{T}\left(t_{i}\right)-R_{A}\left(t_{i}\right)-R_{R}\left(t_{i}\right)-R_{G}\left(t_{i}\right)}{m} ;$

$\left\{\begin{array}{l}\frac{d V\left(t_{i}\right)}{d t} \\ \frac{d x\left(t_{i}\right)}{d t}\end{array}\right\}=\left\{\begin{array}{l}a\left(t_{i}\right) \\ v\left(t_{i}\right)\end{array}\right\}$

donde

$V\left(t_{\mathrm{i}}\right)=V\left(t_{\mathrm{i}-1}\right)+a\left(t_{\mathrm{i}-1}\right) \Delta t \quad \mathrm{y} \quad x\left(t_{\mathrm{i}}\right)=x\left(t_{\mathrm{i}-1}\right)+v\left(t_{\mathrm{i}-1}\right) \Delta t$ 
Verweij (2000) desarrolló un modelo de simulación de velocidad, con el objetivo de calcular la velocidad final de un vehículo pesado en una pendiente ascendente. Verweij (2000) utilizó datos reales de velocidad de un camión instrumentado para obtener perfiles de velocidad como los de la Figura 2. Los resultados de Verweij evidenciaron una importante diferencia con los modelos dinámicos, toda vez que el perfil de velocidad modelado no mostraba con claridad que el vehículo alcanzase la velocidad de equilibrio y la mantuviera en la pendiente. Por el contrario, observó que tendía a producirse una aceleración en el último tercio de la pendiente, como lo muestra la Figura 2.

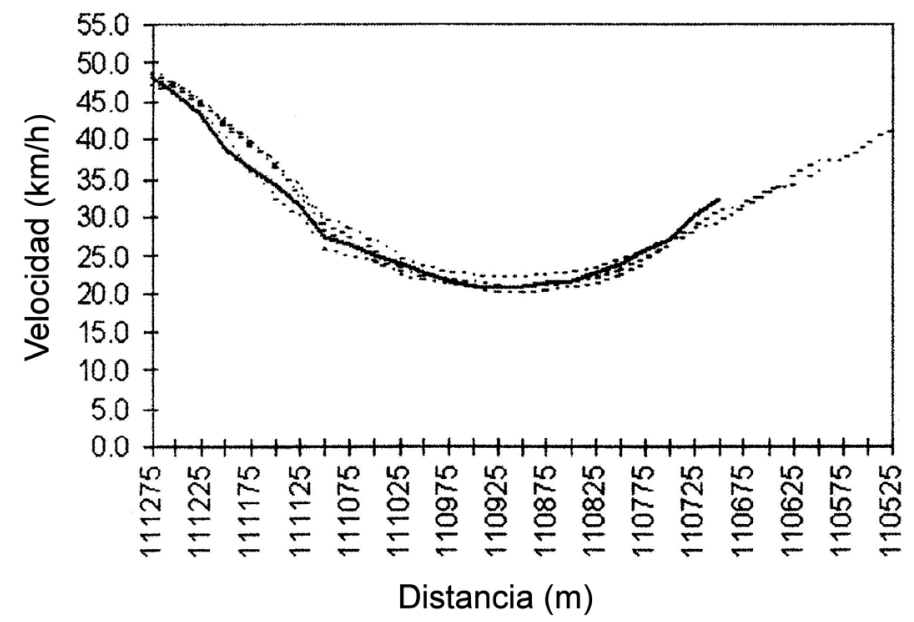

Figura 2: Perfil de velocidad obtenido por Verwij (2000) usando un vehículo pesado instrumentado

\section{Obtención y procesamiento de datos de velocidad}

\section{Diseño factorial}

El diseño factorial tuvo por objeto ordenar y categorizar las variables a medir en terreno, así como identificar en la red vial las rutas en las cuales se obtuvieron los tramos de medición. Para ello se definieron las siguientes categorías de variables:

- Inclinación de la pendiente longitudinal $i$ : A: alta, M: media, B: baja.

- Longitudes de pendientes $L$ : L: largas, M: medias, C: cortas.

- Velocidades de entrada $V_{\mathrm{e}}$ : A: altas, M: medias, B: bajas.

Una de las dificultades de este tipo de estudios es contar con el valor adecuado de peso y potencia nominal de cada vehículo pesado, para determinar adecuadamente la razón $\mathrm{P} / \mathrm{P}$. Por tal motivo, preliminarmente se establecieron categorías provisorias de razón $\mathrm{P} / \mathrm{P}$ en base a las normativas (ver Tabla 1) y a los estudios de Archilla y Fernández de Cieza (1996), Lan y Menendez (2003), Lee y Lee (2000) y Rakha et al. (2001). Con estas categorías para cada variable se determinó el tamaño de la muestra utilizando el método de análisis de potencia estadística aplicado en Echaveguren et al. (2011).

\section{Selección de tramos de medición}

Un tramo de medición se define como un segmento de una red vial que posee pendiente longitudinal sobre una longitud mayor a $200 \mathrm{~m}$. Dicha longitud mínima permite observar los cambios de velocidad a lo largo de toda la pendiente. Para identificarlos se seleccionó de la red vial 6 rutas que contenían tramos de medición de más de 200 m, considerando los siguientes criterios:

- Tipo de vía: pavimentada y bidireccional de 1 o más pistas por sentido,

- volumen de tránsito: tránsito medio diario anual TMDA < 2000 veh/día-año para asegurar condición de flujo libre,

- perfil de elevación: se priorizaron terrenos de topografía ondulada y montañosa,

- restricciones a la velocidad: se descartaron tramos con acceso a centros poblados a que la velocidad está condicionada por la fricción lateral.

Luego de aplicar estos criterios se acotaron los tramos de medición teniendo en cuenta además que:

- El radio de curvas horizontales fuera superior a $500 \mathrm{~m}$, a fin de limitar el efecto de curvatura en la velocidad en la entrada y salida de la pendiente,

- las pendientes longitudinales fueran superiores al $2 \%$ y con longitudes superiores a los $200 \mathrm{~m}$, para lograr representar adecuadamente los cambios de velocidad.

También se caracterizaron las secciones de entrada y salida de la pendiente, con el objetivo de representar la visibilidad en los segmentos anterior y posterior a la pendiente y ver cómo afecta a la velocidad del vehículo. Las características consideradas en las secciones anterior y posterior a la pendiente fueron: recta, curva horizontal e inclinación de pendiente. 
La Figura 3 muestra diagramas de caja con las características geométricas de las pendientes medidas y representadas por el parámetro $\mathrm{CN}=i^{2} L / 1000(i$ en $\% \mathrm{y}$ $L$ en m) que multiplica la pendiente $i$ al cuadrado por la longitud de la pendiente $L$. Se seleccionó este parámetro dado que representa de buena forma la magnitud de las pendientes: pendientes con baja inclinación y baja longitud representan valores bajos de $\mathrm{CN}$ y pendientes prolongadas e inclinadas representan valores altos de CN. En la Figura 3 se aprecia que existen al menos 3 grupos de pendientes en función del parámetro CN. Esta apreciación intuitiva se verificó aplicando análisis de conglomerados usando el algoritmo de k-medias. En la Figura 3 el nivel 1 representa valores de $\mathrm{CN}$ bajo, entre 1 y 16 ; el nivel 2 representa valores intermedios, entre 16 y 31 ; y el nivel 3 representa valores de $\mathrm{CN}$ altos, entre 31 y 48. La mediana de los valores de $\mathrm{CN}$ son, respectivamente, 8, 21 y 40. Estos grupos se utilizan más adelante para discutir los patrones de comportamiento de los perfiles de velocidad.

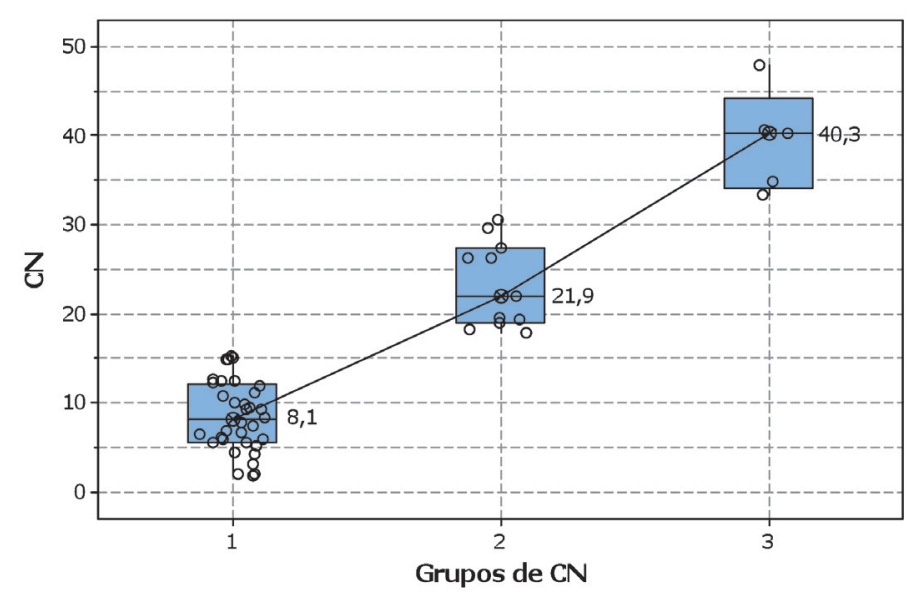

Figura 3: Diagrama de caja con valores de $\mathrm{CN}$ agrupados en 3 niveles

\section{Medición en terreno}

Los datos de velocidad fueron recolectados mediante un GPS dinámico sub-métrico portátil de $10 \mathrm{~Hz}$ instalado en un vehículo liviano, con el cual se realizó seguimiento vehicular. El equipo GPS utilizado captura datos de velocidad cada $0.1 \mathrm{~s}$ con una precisión de $0.1 \mathrm{~km} / \mathrm{h}$ para la velocidad, de $3 \mathrm{~cm}$ para la posición horizontal y de $0.1^{\circ}$ para el azimut. El equipo puede además triangular en movimiento hasta 8 satélites, lo cual le permite obtener una señal estable durante la recolección de datos (Racelogic, 2008). Las principales condiciones de medición fueron: considerar el mismo vehículo para realizar las mediciones; el mismo conductor, previamente entrenado; días de medición con condiciones climáticas similares; distancia entre vehículos estimada visualmente entre 200 y 400 $\mathrm{m}$. Se descartaron mediciones cuando: se producían cambios bruscos de clima; se formaban pelotones; cuando el vehículo líder ejecutaba maniobras que alteraban la continuidad de la circulación (detenciones por ejemplo), el tráfico vehicular era elevado e interfería con las mediciones, obstáculos o trabajos en la vía producían algún tipo de fricción y alteraciones en la velocidad y en las aceleraciones.

\section{Procesamiento de datos}

Los datos se procesaron siguiendo tres pasos: filtrado de las señales de velocidad, corrección de registros de velocidad y suavización de datos de velocidad.

\section{Filtrado de datos de velocidad}

Este filtro de las señales de velocidad permite resolver al mismo tiempo la detección de datos anómalos, recuperar datos perdidos (dropouts) por caída de satélites y acoplar los registros de posición, trayectoria y velocidad obtenidos con el GPS. Para filtrar registros de velocidad se utilizó el filtro de Kalman provisto por el software de procesamiento de datos del dispositivo GPS utilizado en las mediciones, de acuerdo a las recomendaciones de uso descritas en Racelogic (2008).

\section{Corrección de datos de velocidad}

El ajuste por seguimiento vehicular se realizó para deducir la velocidad del vehículo líder a partir de la velocidad medida con el vehículo instrumentado (vehículo seguidor), mediante simulación. La expresión (5) corresponde al modelo de Gaziz et al. (1961) integrado al modelo de Wolshon y Hatipkarasulu (2000) con los coeficientes calibrados por Che-Puan (2004).

$$
\Delta V(t)=\frac{a_{2}\left(t^{\prime}\right)}{V_{2}\left(t^{\prime}\right)}\left[4.4+1.12 V_{2}\left(t^{\prime}\right)\right]
$$

En donde $\Delta V(t)$ representa la diferencia de velocidad entre el vehículo líder y el seguidor en el instante de tiempo $t$. $a_{2}\left(t^{\prime}\right)$ y $V_{2}\left(t^{\prime}\right)$ son los vectores de aceleración y velocidad respectivamente del vehículo seguidor. $t^{\prime}=t+T$, con $T$ 
el tiempo de reacción fijado en $1.6 \mathrm{~s}$. Los vectores $a_{2}\left(t^{\prime}\right)$ y $V_{2}\left(t^{\prime}\right)$ se obtuvieron directamente a partir de señales de velocidad registradas por el GPS. Al aplicar el modelo se obtuvo un valor de raíz cuadrática media (RSME) entre 1 y $2.2 \mathrm{~km} / \mathrm{h}$, lo cual representa el error de estimación indirecta de velocidad mediante seguimiento vehicular. Considerando que las velocidades varían entre 30 y 100 $\mathrm{km} / \mathrm{h}$, el valor de RMSE obtenido se considera muy bueno. La Figura 4 muestra un ejemplo de perfiles de velocidad medidos y corregidos mediante este método.

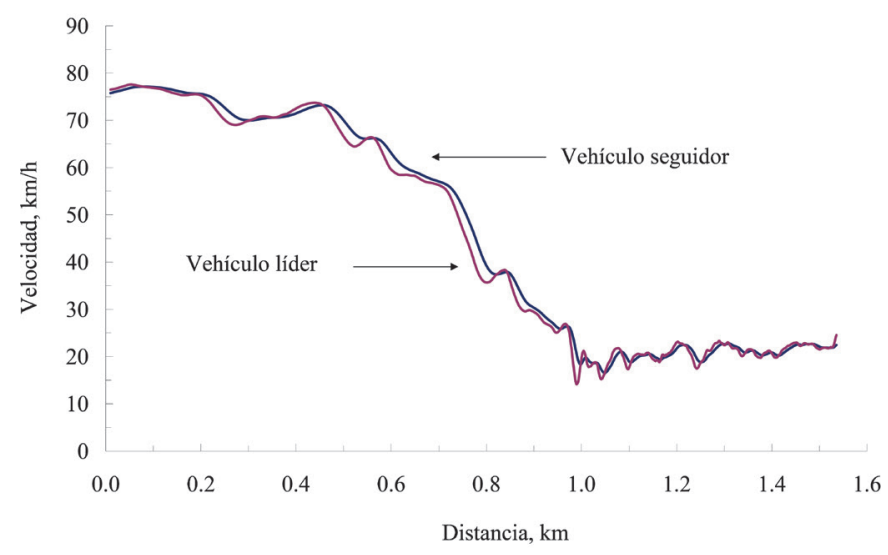

Figura 4: Ejemplo de perfiles de velocidad medidos y corregidos

\section{Suavización de datos de velocidad}

Después de filtrar y corregir los datos se realizó la suavización, con el fin de reducir el ruido en cada perfil de velocidad y simplificar así su interpretación. Existen diversos métodos de suavización, tales como la regresión local cruzada, la suavización en base a kernel, suavizadores exponenciales dobles y simples, splines de orden $n$ y otras más sofisticadas basadas en inteligencia artificial y wavelets (Echaveguren et al., 2013). Después de un examen exploratorio de estas técnicas, se optó por utilizar el suavizador Loess (suavización de datos ponderados localmente). Este suavizador permite obtener mediante regresión no paramétrica alrededor del punto de datos $x_{\mathrm{i}}$, una estimación de la función de datos discretos $f\left(x_{\mathrm{i}}\right)$, asumiendo que el ruido $\varepsilon_{\mathrm{i}}$ se distribuye normal con media 0 y desviación estándar $\sigma$, según la expresión (6).

$y_{\mathrm{i}}=f\left(x_{\mathrm{i}}\right)+\varepsilon_{\mathrm{i}}$

El método aplica regresión de mínimos cuadrados ponderados en una ventana de datos $h$ que se desplaza a lo largo de la señal de velocidad para cada punto de referencia $x_{i}$. La función de ponderación es la función tri-cúbica descrita en la expresión (7), en donde $x_{0}$ es el dato alrededor del cual se define el valor de $h$. La Figura 5 muestra un ejemplo de un perfil de velocidad suavizado superpuesto al perfil de velocidad medido, filtrado y corregido.

$w_{i}=\left\{\begin{array}{c}\left(1-\left|\frac{x_{i}-x_{0}}{h}\right|^{3}\right)^{3} ; \quad\left|\frac{x_{i}-x_{0}}{h}\right|<1 \\ 0 \quad ; \quad \frac{x_{i}-x_{0} \mid}{h} \mid \geq 1\end{array}\right.$

De este modo, el perfil de velocidad con el que se aplica el análisis queda libre de datos anómalos, con los registros de velocidad y posición acoplados y sin el ruido producido por pequeñas aceleraciones locales. Con este procedimiento, se generaron 51 perfiles de velocidad válidos para el análisis, para un rango de pendientes entre el 2 y $8 \%$ sobre longitudes entre 200 y $2400 \mathrm{~m}$.

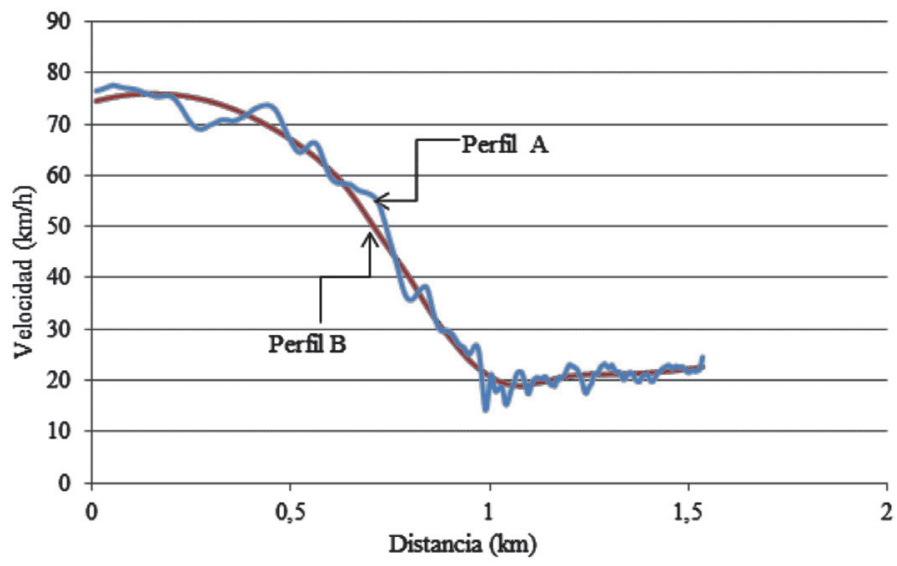

Figura 5: Ejemplo de perfil de velocidades suavizado superpuesto sobre el original

\section{Resumen de perfiles de velocidad procesados}

Una vez aplicados los procesos de depuración, filtrado, ajuste y suavizado, se obtuvieron 51 perfiles de velocidad para posterior análisis. El perfil de velocidad se puede caracterizar con 4 valores de velocidad: La velocidad de entrada a la pendiente $V_{\mathrm{i}}$, la velocidad máxima en la pendiente $V_{\max }$, la velocidad mínima en la pendiente $V_{\min }$ y la velocidad de salida de la pendiente $V_{\mathrm{f}}$.

\section{Análisis de perfiles de velocidad}

\section{Comportamiento de la velocidad}

En un perfil de velocidad ideal, la velocidad de entrada 
a la pendiente coincide con la velocidad máxima en la pendiente, y la velocidad mínima coincide con la velocidad de salida de la pendiente. Esta última corresponde a la velocidad de equilibrio: aquella velocidad en la cual las fuerzas resistivas al movimiento y las fuerzas tractrices se igualan, resultando en una aceleración nula. En las siguientes secciones se analizan estas 4 velocidades.

\section{Velocidad de entrada}

La velocidad de entrada a la pendiente depende de las condiciones del diseño anterior. Si la pendiente está precedida de una recta, la velocidad de entrada será superior a la que se logre en presencia de curvas, restricciones de velocidad u otra. Por tanto, es previsible que exista variabilidad en la velocidad de entrada. En las mediciones realizadas, se consideraron curvaturas bajas en la entrada, de manera de visualizar la variabilidad en la velocidad de entrada en las condiciones más favorables. La Figura 6 muestra el diagrama de frecuencia acumulada de la velocidad de entrada, asociado a una distribución de probabilidades normal, con media igual a $85 \mathrm{~km} / \mathrm{h}$ y desviación estándar de $11 \mathrm{~km} / \mathrm{h}$. El test de AndersonDarling y el valor $p$ muestran que el ajuste a la distribución normal es significativo.

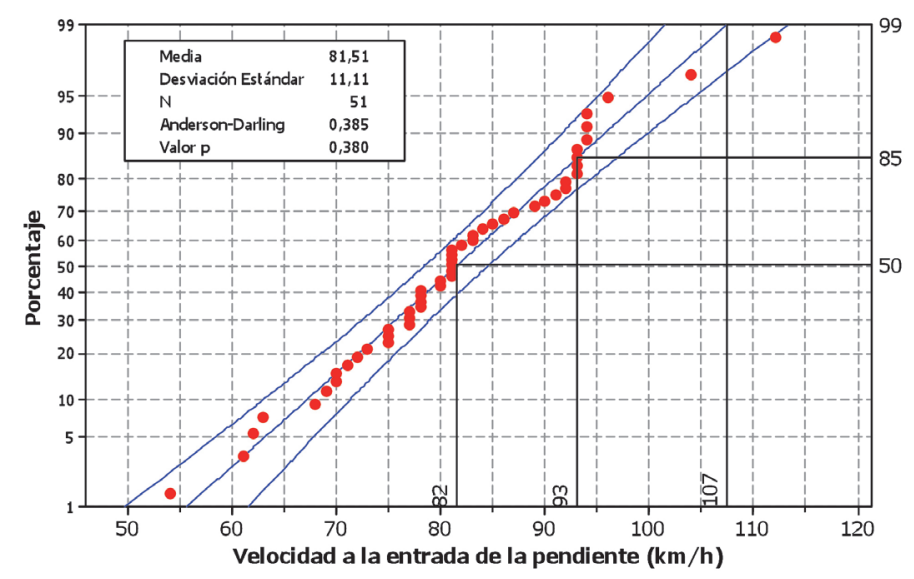

Figura 6: Distribución de frecuencia acumulada de velocidades de entrada medidas en terreno

El percentil 50 de la Figura 6 representa el valor de velocidad media y posee un valor de $82 \mathrm{~km} / \mathrm{h}$. El percentil 85 representa la velocidad de operación. El valor obtenido fue de $93 \mathrm{~km} / \mathrm{h}$. El percentil 99 representa al conductor más rápido que ingresa a la pendiente. Notar que la velocidad de ingreso a las pendientes definidas por el Manual de
Carreteras de Chile (MOP, 2010), de $88 \mathrm{~km} / \mathrm{h}$, equivale aproximadamente al percentil 72 de la distribución, es decir, se encuentra bajo la velocidad de operación.

\section{Velocidad de salida}

La velocidad de salida depende de la geometría de la pendiente. Por tanto, en la medida que $\mathrm{CN}$ varíe, la velocidad de salida también variará. Por tal motivo las distribuciones de frecuencia acumulada no son únicas. Teniendo en cuenta la agrupación de valores de CN, la Figura 7 muestra los diagramas de frecuencia acumulada para $\mathrm{CN}$ alto, intermedio y bajo (Grupo 1, 2 y 3 respectivamente). En la Figura 7 se puede apreciar que en la medida que $\mathrm{CN}$ es más bajo, la velocidad de operación a la salida es más alta. Esto es consistente con lo que intuitivamente se espera: cuando la pendiente es más elevada y de mayor longitud, existe una mayor pérdida de velocidad. En este caso, la velocidad de operación representada por el percentil 85 , pasa de $80 \mathrm{~km} / \mathrm{h}$ a $69 \mathrm{~km} / \mathrm{h}$ y luego a $58 \mathrm{~km} / \mathrm{h}$ en la medida que $\mathrm{CN}$ aumenta.

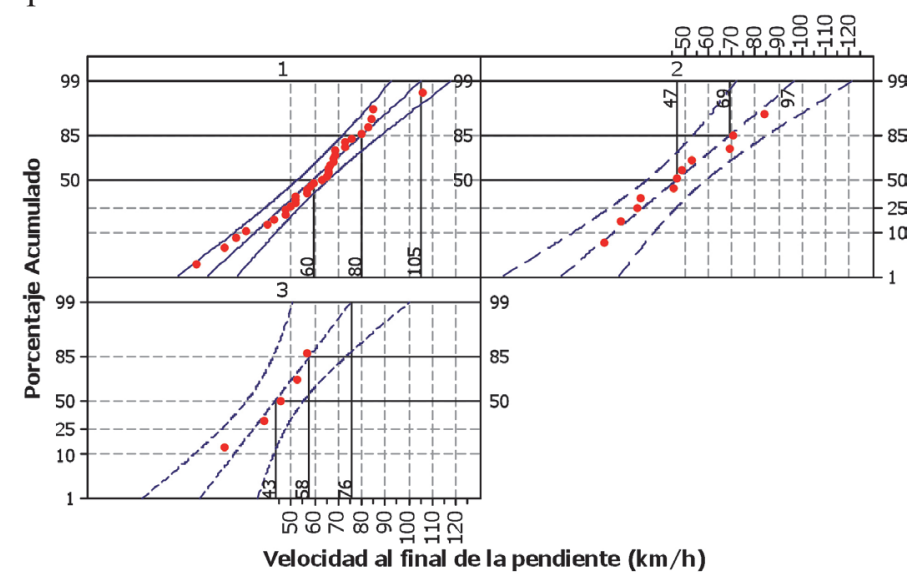

Figura 7: Distribución de frecuencia acumulada de velocidades de salida medidas en terreno y agrupadas según el valor de $\mathrm{CN}$

\section{Pérdida de velocidad en la entrada de la pendiente}

La pérdida de velocidad corresponde a la diferencia entre la velocidad de entrada $V_{\mathrm{i}}$ y la velocidad mínima $V_{\min }$ en la pendiente. La Figura 8 muestra esta pérdida con respecto a cada una de las categorías geométricas de la pendiente para cada uno de los grupos de CN. La Figura 8 muestra que la pérdida de velocidad varía desde $26 \mathrm{~km} / \mathrm{h}$ para $\mathrm{CN}$ bajos hasta $46 \mathrm{~km} / \mathrm{h}$ para valores de $\mathrm{CN}$ elevados. Esta diferencia es muy superior al umbral de $15 \mathrm{~km} / \mathrm{h}$ establecido por AASHTO (2011) para definir la necesidad de emplazar pistas auxiliares. 


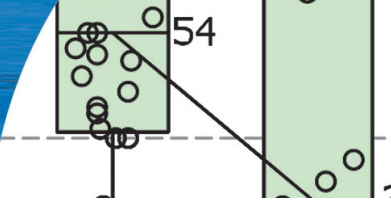

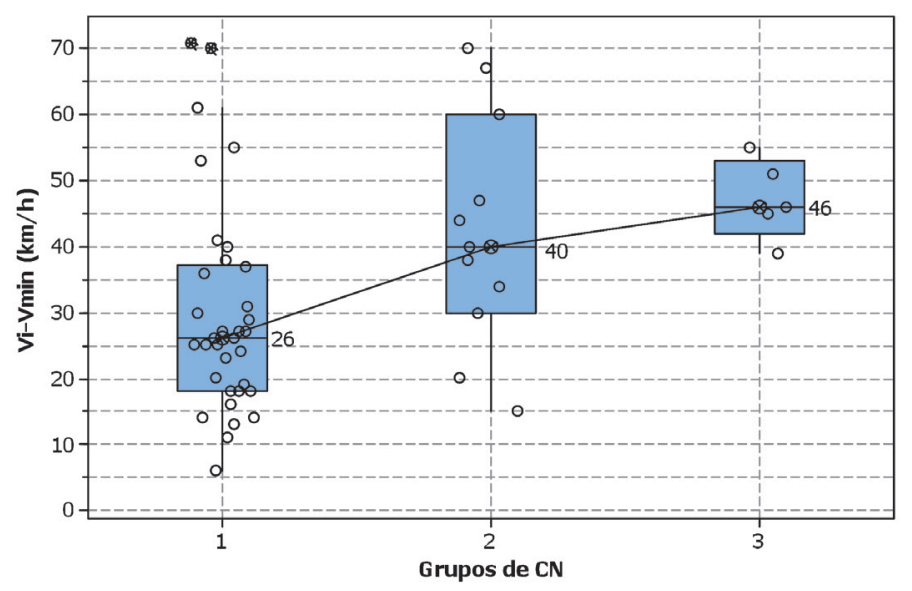

Figura 8: Pérdida de velocidad en la pendiente para cada grupo de $\mathrm{CN}$

\section{Ganancia de velocidad en la salida de la pendiente}

Al contrario de lo que resulta de los modelos, en terreno se observó una ganancia de velocidad en el último tramo de la pendiente. Esta ganancia se grafica en la Figura 9 mediante diagramas de caja de la velocidad mínima $V_{\min }$ y de la velocidad de salida de la pendiente $V_{\mathrm{f}}$, para cada grupo de $\mathrm{CN}$. Se puede ver que en el grupo 1 de $\mathrm{CN}$ que la ganancia de velocidad, expresada como la diferencia entre $V_{\mathrm{f}}$ menos $V_{\min }$ es $10 \mathrm{~km} / \mathrm{h}$. En el grupo 2 la ganancia es de $16 \mathrm{~km} / \mathrm{h}$ y en el grupo $3 \mathrm{de} 19 \mathrm{~km} / \mathrm{h}$. A partir de estos resultados se puede deducir que el perfil observado difiere en forma de los perfiles predichos por los modelos y se acerca más a los resultados empíricos obtenidos por Verweij.

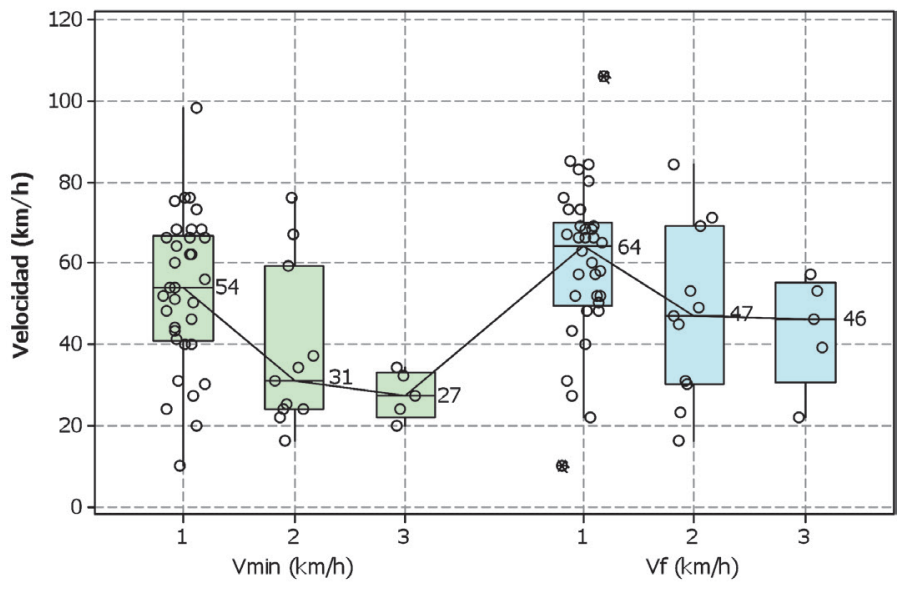

Figura 9: Diagramas de caja de velocidad mínima en la pendiente y la velocidad de salida de la pendiente según grupos de $\mathrm{CN}$

\section{Análisis de la forma del perfil}

Los resultados descritos en la sección anterior permiten identificar un patrón de comportamiento de cada perfil de velocidad, el cual se puede sintetizar en dos factores de forma F1 y F2 de acuerdo a la expresión (8).

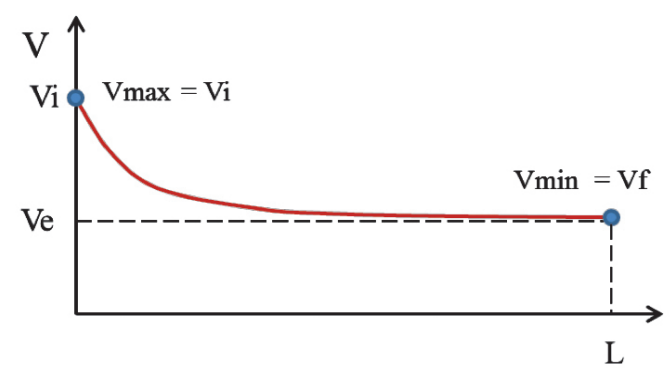

a) $\mathrm{F} 1=1 ; \mathrm{F} 2=1$

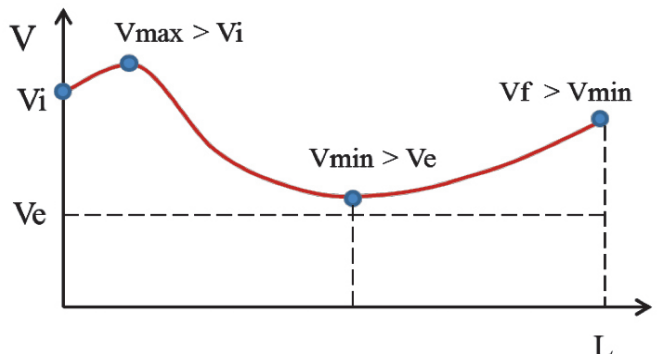

c) F1 $<1 ;$ F2 $>1$

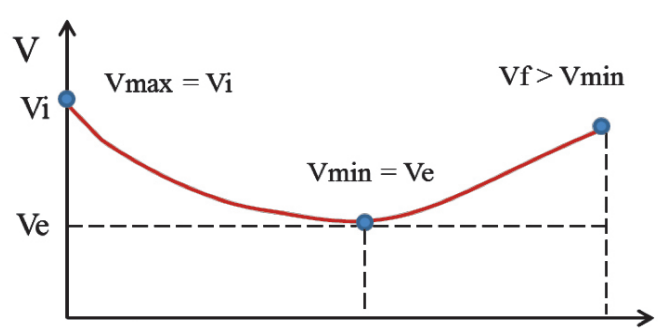

$\mathrm{L}$

b) $\mathrm{F} 1=1 ; \mathrm{F} 2<1$

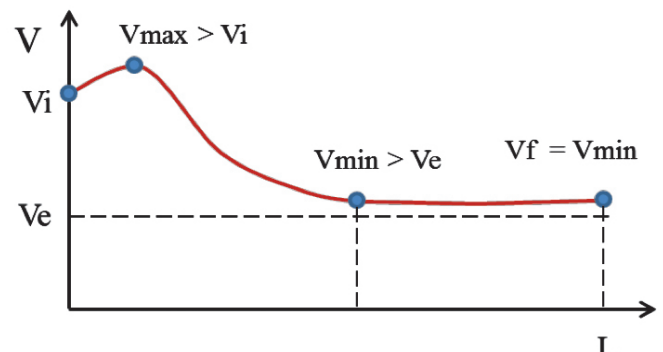

d) $\mathrm{F} 1<1 ; \mathrm{F} 2=1$

Figura 10: Factores de forma para diversos perfiles de velocidad 


$$
\mathrm{F} 1=\frac{V_{i}}{V_{\max }} ; \quad \mathrm{F} 2=\frac{V_{\text {min }}}{V_{f}}
$$

La Figura 10 ilustra casos típicos de factores de forma identificados en terreno. En todas ellas $L$ representa la longitud de la pendiente. La Figura 10a muestra el caso de un perfil ideal en el cual la velocidad máxima $V_{\max }$ coincide con la velocidad de entrada $V_{\mathrm{i}}(\mathrm{F} 1=1)$ y la velocidad de equilibrio $V_{\mathrm{e}}$ coincide con la velocidad mínima $V_{\min }$ y la velocidad de salida de la pendiente $V_{\mathrm{f}}(\mathrm{F} 2=1)$. Este es el tipo de modelo que se utiliza en la mayoría de las normas de diseño. La Figura 10b muestra un perfil similar en forma al obtenido por Verwij (2000). Las Figuras 10c y 10d representan otras posibles formas de perfiles de velocidad, de acuerdo a los datos obtenidos en terreno en este estudio.

Se calcularon los factores de forma para todos los perfiles medidos en terreno. Los resultados se sintetizan en la Figura 11, que muestra para cada grupo de $\mathrm{CN}$ los factores de forma F1 y F2 calculados para las 51 pendientes. En la Figura 11 se aprecia que el factor de forma F1 varía entre 0.95 y 0.99 para los tres grupos de CN. Por tanto, la velocidad de entrada a la curva es efectivamente similar a la velocidad máxima en la pendiente. Para el caso de F2 la situación es diferente. El factor de forma F2 $<1$ muestra que existe una ganancia de velocidad en el $75 \%$ de los casos y que esta ganancia de velocidad es mayor en la medida que la pendiente es más pronunciada y de mayor longitud, caso correspondiente al grupo de valores 3 de CN.

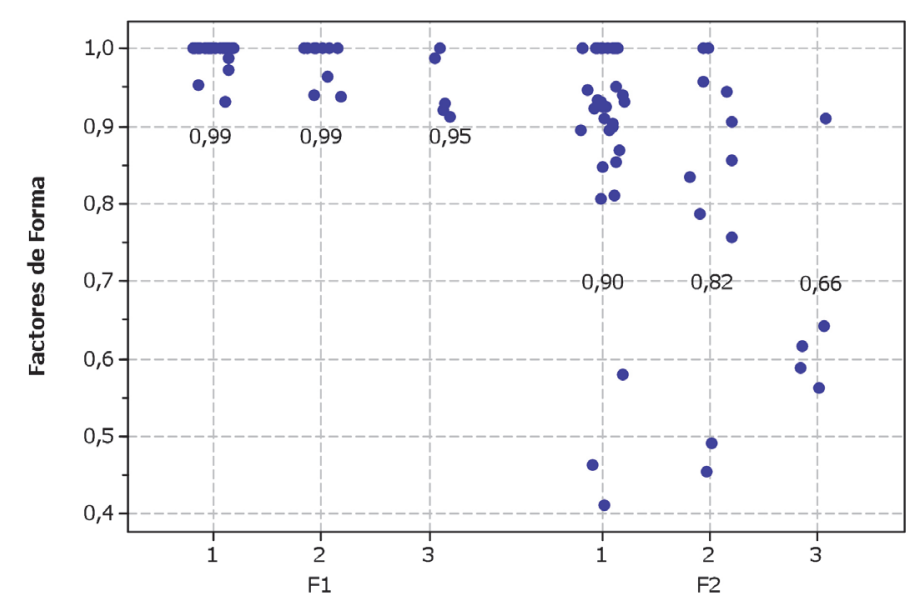

Figura 11: Factores de forma de los perfiles de velocidad procesados para distintos valores de $\mathrm{CN}$
Asumiendo que un valor aceptable para F1 y F2 es $1.0 \pm$ 0.05 , se tiene que:

- El $56 \%$ de los perfiles medido se asemejan en forma a los modelos teóricos

- En el 14\% de los perfiles se observó que la velocidad de entrada corresponde a la velocidad máxima (F1 = $1.0 \pm 0.05)$

- En el $68 \%$ de los perfiles se observó una ganancia de velocidad

\section{Cálculo de relación peso/potencia}

Se seleccionaron los perfiles de velocidad sin distorsiones por ganancia de velocidad. De estos perfiles se seleccionó la pendiente, longitud, velocidad inicial y velocidad de salida. Con estos datos, se utilizó el modelo de Rakha et al. (2001), para obtener por retro-cálculo la relación $P P$ efectiva de los vehículos pesados medidos en terreno. La Tabla 2 muestra los resultados obtenidos.

Los valores de $P P$ se pueden agrupar en 3 conglomerados de 106 a $199 \mathrm{~kg} / \mathrm{kW}$ de 230 a $274 \mathrm{~kg} / \mathrm{kW}$ y de 378 a 429 $\mathrm{kg} / \mathrm{kW}$. Sin embargo, los valores usuales de relaciones peso/potencia para vehículos pesados de unidad simple y articulados es de hasta $150 \mathrm{~kg} / \mathrm{kW}$. Valores superiores de la relación peso/potencia corresponden a configuraciones de alto tonelaje, que para el cálculo de la potencia media se consideran como datos anómalos.

Los valores de velocidad de equilibrio se calcularon con la expresión de Lan y Menendez (2003), ver expresión (9). En esta expresión la eficiencia del motor y la resistencia al rodado son variables de entrada al modelo. Para el cálculo, se adoptó una eficiencia del motor $\eta$ del 95\% y una resistencia al rodado $f_{\text {rc }}$ de 0.0139 . Los resultados de la Tabla 2 muestran que la velocidad de equilibrio $V_{\mathrm{e}}$ calculada, es sistemáticamente inferior a la velocidad mínima $V_{\min }$. En promedio, la velocidad mínima resultó 1.2 veces superior a la velocidad de equilibrio para las relaciones peso/potencia $P P$ retro calculadas.

$$
V_{\text {equilibrio }}=367.35 \frac{\eta}{P P\left(f_{r c}+i\right)}
$$


Tabla 2: Características de perfiles utilizados para el cálculo de la relación peso/potencia

\begin{tabular}{|c|c|c|c|c|c|c|}
\hline $\begin{array}{c}\text { Perfil } \\
\mathrm{N}^{\mathbf{0}}\end{array}$ & $i, \%$ & $L, \mathrm{~m}$ & $\begin{array}{c}V_{\mathrm{i}}, \\
\mathrm{km} / \mathrm{h}\end{array}$ & $\begin{array}{c}V_{\min }, \\
\mathrm{km} / \mathrm{h}\end{array}$ & $\begin{array}{c}V_{\mathrm{e}}, \\
\mathrm{km} / \mathrm{h}\end{array}$ & $\begin{array}{c}P P, \\
\mathrm{~kg} / \mathrm{kW}\end{array}$ \\
\hline 6 & 2.9 & 516 & 77 & 66 & 58 & 140 \\
\hline 7 & 4.0 & 697 & 63 & 27 & 24 & 274 \\
\hline 11 & 2.4 & 1122 & 68 & 43 & 37 & 250 \\
\hline 17 & 2.7 & 1640 & 93 & 68 & 58 & 146 \\
\hline 27 & 4.6 & 703 & 85 & 48 & 43 & 137 \\
\hline 28 & 2.2 & 414 & 92 & 31 & 26 & 378 \\
\hline 41 & 3.3 & 540 & 75 & 20 & 17 & 429 \\
\hline 43 & 4.1 & 746 & 83 & 54 & 48 & 133 \\
\hline 44 & 2.8 & 708 & 81 & 75 & 65 & 129 \\
\hline 46 & 3.8 & 680 & 87 & 64 & 57 & 119 \\
\hline 48 & 3.6 & 949 & 94 & 68 & 60 & 117 \\
\hline 49 & 3.7 & 379 & 93 & 66 & 59 & 117 \\
\hline 52 & 4.1 & 415 & 92 & 62 & 55 & 116 \\
\hline 55 & 2.2 & 378 & 94 & 76 & 65 & 150 \\
\hline 56 & 4.2 & 539 & 86 & 66 & 59 & 106 \\
\hline 58 & 3.7 & 566 & 71 & 52 & 46 & 150 \\
\hline 60 & 3.8 & 587 & 77 & 50 & 44 & 153 \\
\hline 61 & 1.9 & 544 & 69 & 56 & 46 & 230 \\
\hline 65 & 3.9 & 442 & 78 & 60 & 53 & 124 \\
\hline 66 & 4.5 & 463 & 78 & 40 & 35 & 167 \\
\hline 70 & 3.0 & 673 & 80 & 54 & 47 & 169 \\
\hline
\end{tabular}

\section{Comparación con normativa Chilena}

La normativa Chilena utiliza un perfil de velocidad estandarizado adaptado del Manual de Capacidad de Carreteras (TRB, 2000). Puesto que el modelo de la normativa Chilena se expresa en un gráfico, fue necesario construir un modelo numérico en base al modelo de Rakha et al. (2001) a fin de reconstituir los perfiles de velocidad. Se consideró para ello un rango de pendientes entre el 1 y el $8 \%$, una longitud entre 0 y $4200 \mathrm{~m}$ y una velocidad de entrada de $88 \mathrm{~km} / \mathrm{h}$. Para lograr el ajuste del modelo al utilizado en la normativa se consideró un vehículo pesado con una razón peso/potencia entre 144 y $210 \mathrm{~kg} / \mathrm{kW}$. Con estos datos se logró replicar el modelo graficado en el Manual de Carreteras de Chile (MOP, 2010). Posteriormente se seleccionaron del total de perfiles de la Tabla 2 aquellos en que la razón $P P$ se encontraba dentro del rango en el cual se construyó el perfil de velocidad. Estos se graficaron en conjunto con los perfiles de velocidad de la normativa de Chile, como lo muestra la Figura 12. En la Figura 12 se aprecia que los patrones generales de comportamiento de los perfiles de velocidad medidos son similares para pendientes bajas en el primer tramo de la pendiente. Existe un desfase de velocidad inicial $V_{\mathrm{i}}$ con respecto al perfil de velocidad de la normativa debido a que ésta sólo usa un valor de velocidad inicial. Puesto que la velocidad de entrada a la pendiente depende de las condiciones geométricas del trazado anterior a la pendiente, el usar un solo valor de velocidad inicial no resulta adecuado.

Los perfiles 6, 55, 58 y 60 están asociados a una razón peso/ potencia de alrededor de $150 \mathrm{~kg} / \mathrm{kW}$, valor de potencia que es distinto al asignado al vehículo tipo usado para construir el perfil de velocidad de la normativa. Esto marca una diferencia relevante al momento de modelar el perfil de velocidad bajo principios mecanicistas.

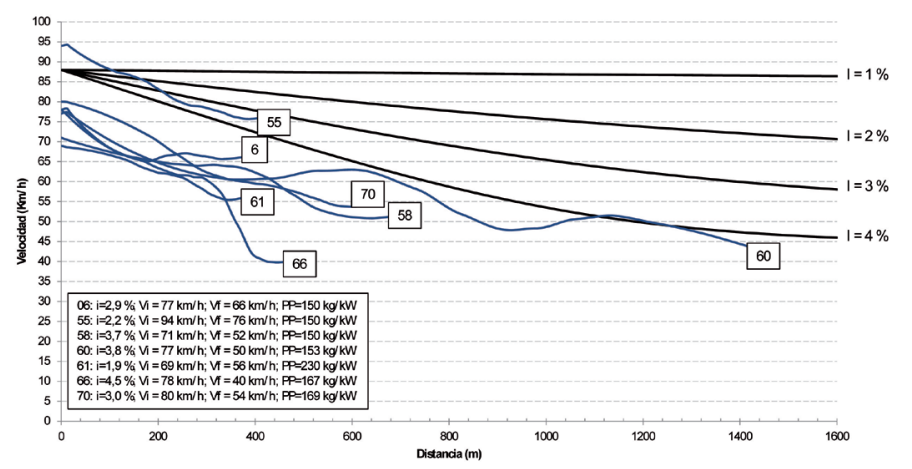

Figura 12: Comparación de datos empíricos con perfil de velocidad de la normativa Chilena

\section{Conclusiones}

Este artículo discute el patrón de comportamiento de perfiles de velocidad de vehículos pesados en pendientes ascendentes, en base a datos obtenidos en terreno. Se realizaron 70 mediciones de perfiles de velocidades en 6 rutas del sur de Chile mediante el método de seguimiento vehicular. Se utilizó un GPS de alta precisión que entrega datos discretos cada $0.1 \mathrm{~s}$, lo que hace posible tener una visión global del comportamiento de la velocidad del camión en la pendiente. Luego del procesamiento de datos, se obtuvieron 51 perfiles de velocidad válidos para realizar el análisis. En este trabajo se consideraron las pendientes completas, y no sólo la parte homogénea de la pendiente, por lo cual el perfil de velocidad incluye los cambios de velocidad al inicio y fin de pendiente, producto 
de las variaciones paulatinas de inclinación a la entrada y salida de la pendiente inducidos por curvas verticales. La velocidad de entrada a la pendiente está condicionada por la condición del trazado precedente. En terreno se verificó que la velocidad de entrada se distribuye normal, y que el percentil 85 , correspondiente a la velocidad de operación, es superior al valor de velocidad empleado en la normativa de Chile. En este sentido, parece razonable aumentar la diversidad de posibilidades de velocidad de entrada a la pendiente considerando por ejemplo velocidades asociadas a terreno plano, ondulado y montañoso.

La velocidad de salida por el contrario, está condicionada por la longitud e inclinación de la pendiente, pero también por el peso/potencia del vehículo pesado. En terreno se observó que el $68 \%$ de los perfiles mostraba una ganancia de velocidad en el último tercio de la pendiente, resultado consistente con las observaciones de Verweij. Esto quiere decir que a la velocidad mínima se da que la fuerza tractriz del vehículo en combinación con su peso, es superior a las fuerzas resistentes, quedando un remanente de potencia para acelerar, lo cual lleva a que el vehículo no alcance la velocidad de equilibrio, particularmente en pendientes con valores de $\mathrm{CN}$ bajos.

En base a la revisión de los perfiles obtenidos en terreno y a la literatura revisada, se concluye además que los fenómenos de transición de velocidad en la entrada y salida de las pendientes no están incluidos en los modelos físicos de la ecuación de equilibrio dinámico, por lo cual las condiciones iniciales no están debidamente incluidas en los modelos existentes. Asimismo, el efecto sobre la liberación de potencia en transiciones de pendientes heterogéneas es un fenómeno que no está suficientemente estudiado en los modelos, y que explica en parte las oscilaciones de velocidad observadas en los perfiles. Por lo tanto es necesario re-formular los modelos de cálculo de perfil de velocidad para incluir estos patrones de comportamiento en los modelos y aproximar de mejor manera los modelos al comportamiento empírico.

Una de las limitaciones de este trabajo fue el no contar con una estimación más precisa de la relación peso/potencia, debido a que no se contó en la investigación con plazas de pesaje móvil para obtener los datos de los vehículos seguidos. Por tal motivo se optó por realizar un retro cálculo en base a velocidades mínimas en perfiles similares a los que establecen los modelos teóricos.

Otro aspecto no considerado fue la estimación en terreno de headway geo-referenciado producido entre el vehículo líder y el seguidor, lo cual permitiría una mejor estimación del error de medición de velocidad con el método de seguimiento vehicular. Por tal motivo se optó por realizar simulación de error en base a datos de terreno y modelos calibrados de brechas, con el fin de contar con un orden de magnitud del error de la medición realizada con el mecanismo de seguimiento vehicular. Se obtuvo un error medio entre 1 y $2.2 \mathrm{~km} / \mathrm{h}$, el cual se considera satisfactorio, que puede mejorarse mediante mediciones de headway.

\section{Referencias}

AASHTO (2011). A Policy on Geometric Design of Highways and Streets. American Association of State Highway and Transportation Officials, USA

Archilla, A. and Fernández de Cieza, A. (1996). Truck performance on Argentinean highways. Transportation Research Record 1555, $114-123$

AUSTROADS (2009). Guide to Road Design Part 3: Geometric Design. Association of Australian and New Zealand Road Transport and Traffic Authorities, Australia

Che-Puan, O. (2004). Driver's car following headway on single carriageway roads. Journal Kejuruteraan Awam 16(2), 15 - 27

DGC (1999). Norma 3.1-IC: Características geométricas: Trazado. Dirección General de Carreteras. Ministerio de Fomento, España

Echaveguren T. y Vargas, S. (2007). Metodología de análisis y diseño de lechos de frenado. Revista de Ingeniería de Construcción 22(3), 175 - 184

Echaveguren, T., Sepúlveda, P. y Vargas-Tejeda, S. (2011). Evaluación de precisión de mediciones de velocidad de operación en carreteras obtenidas con GPS. XV Congreso Chileno de Ingeniería de Transporte, Artículo \#91, Santiago, Chile

Echaveguren, T., Tudela, A. and Fonseca, C. (2013). Assessment of smoothing techniques applied to speed profiles measured with GPS RTK. 13th World Conference on Transport Research, Rio de Janeiro, Brasil

Fitch, J.W. (1994). Motor truck engineering handbook. 4th ed. SAE, USA 
Gaziz, D.C., Herman, R. and Rothery, R.W. (1961). Nonlinear follow-the-leader models of traffic flow. Operations Research 9(4), $545-567$

Gillespie, T.D. (1985). Methods for predicting truck speed loss on grades. Report UMTRI - 85 - 39/1. University of Michigan Transport Research Institute, USA

INVIAS (2008). Manual de Diseño Geométrico, Instituto Nacional de Vías. Ministerio de Transportes. Colombia

Lan, Ch-J. and Menendez, M. (2003). Truck speed profile models for critical length of grade. Journal of Transportation Engineering 129(4), 408 - 419

Lee, S. and Lee, D. (2000). Validation of the 10 MHP rule in highway design consistency procedure. Proceedings of 2 nd International Symposium on Highway Geometric Design, Germany, $364-376$

MOP (2010). Instrucciones y Criterios de Diseño. Manual de Carreteras, Volumen 3. Ministerio de Obras Públicas, Chile

Racelogic (2008). VBOX Mini User Guide. UK

Rakha, H., Lucic, I., Demarchi, S.H., Setti, J.R. and Van Aerde, M. (2001). Vehicle dynamics model for predicting maximum truck acceleration levels. Journal of Transportation Engineering 127(5), 418-425
Rakha, H. and Lucic, I. (2002). Variable power vehicle dynamics model for estimating truck accelerations. Journal of Transportation Engineering 128(5), 412 - 419

Rakha, H. and Yu, B. (2004). Truck performance curves reflective of truck and pavement characteristics. Journal of Transportation Engineering 130(6), 753 - 767

Verweij, C.A. (2000). Evaluating truck speeds on vertical alignments. Proceedings of 2nd International Symposium on Highway Geometric Design, Germany, 486 - 498

TRB (2000). Highway Capacity Manual, Transportation Research Board, Washington D.C. USA

Wong, J.Y. (2001). Theory of ground vehicles. 3rd ed. Wiley, New York

Wolshon, B. and Hatipkarasulu, Y. (2000). Results of car following analyses using global positioning system. Journal of Transportation Engineering 126(4), 324 - 331 